\title{
Zur Kenntnis der optischen Eigenschaften disperser Systeme.
}

\section{Ueber Farbenänderungen durch Belichtung.}

\author{
Von Fritz Weigert (Leipzig?.
}

(Eingegangen am (-. Januar 1921.)

\section{$\$ 1$. Einleitung.}

Die Untersuchung der optischen Eigenschaften chemischer Systeme, speziell der spektralen Verteilung der Lichtabsorption, ist das feinste Hilfsmittel zur Erkenntnis ihres Aufbaus. Dieses Hilfsmittel ist auf disperse Systeme schon vielfach angewendet worden, und die experimentelle und theoretische Untersuchung ihrer Farben und Farbänderung bei verschiedener Herstellung und Nachbehandlung hat zahlreiche charakteristische Eigenschaften der Kolloide erschlossen. Man hat daraus gelernt, daB das Absorptionsspektrum disperser Systeme, bei Ausschlub chemischer Veränderungen, in einem nahen Zusammenhang mit dem Dispersitätsgrad des Systems und den optischen Eigenschaften der einzelnen Molekülarten steht, aus welchen die disperse Phase und das Dispersionsmittel aufgebaut sind.

Der Dispersitătsgrad kann bei fast allen diesen Versuchen nur auf ziemlich grobem und unübersichtlichem Wege durch Variation bei der Herstellung des kolloiden Systems geändert werden. Ebenso ist man noch nicht imstande, mit Sicherheit bestimmte Zustände, z. B. ein Silbersol von genau definierter Farbe, zu reproduzieren, weil der Dispersitătsgrad mit einer Reihe verschiedener Paktoren in einem $\mathrm{Zu}$ sammenhang steht, der noch nicht vollkommen erkannt ist. Die Schlüsse, welche man von den optischen Eigenschaften eines Systems auf den Dispersitätsgrad, und umgekehrt vom Dispersitatsgrad auf das $\Lambda$ bsorptionsspektrum zieht, sind daher noch ziemlich unsichere.

Wohl der einzige Fall, bei dem es möglich ist, die Farben kolloider Systeme auf einem einfachen und übersichtlichen Wege reversibel zu verändern, ist die zuerst von Faraday') gemachte Beobachtung, daß die Farbe von Goldgelatine in trocknem und feuchtem Zustande verschieden ist. Diese Erscheinung wurde von $\mathrm{Ki}$ ichner und Zsigmond ${ }^{2}$ ) an Silber- und Goldgelatinepräparaten sehr eingehend unter-' sucht und in der Weise gedeutet, dab durch den Wasser-Eintritt und -Austritt in dem System die Lagerungsdichte der einzelnen Metallkomplexe reversibel geändert wird, was eine rever-

1) W. Far a day, Phil. Trans. 147, 175 (1857).

2) F. Kirchner u. R. Zsig mondy, Ann. d. Phys. 18, 573 (1904). sible Farbeänderung zur Folge hat. Aber auch in diesem Fall sind die Beziehungen zwischen Ursache und Wirkung nicht eindeutig: Es findet eine Volumenvergrößerung bei der Quellung der trocknen Gelatine durch Wasseraufnahme statt, das Wasser tritt als never Stoff in das System ein, der Brechungsexponent und die Oberflächenspannung ändern sich, so dab es noch unentschieden ist, welche Veränderungen des Systems direkt mit der Farbenänderung verknüpft sind.

Es ist zu eiwarten, daB man über den $\mathrm{Zu}$ sammenhang zwischen dem Zustand eines kolloiden Systems und seiner Lichtabsorption etwas mehr erfährt, wenn man imstande ist. es auf mildere und differenziertere Weise zu verändern, als durch die erwähnten Methoden. Dies scheint nun auf Grund einer neuen Beobachtung möglich $z u$ sein, welche ich vor kurzer Zeit beschrieben habe. Es ist dies die überraschende Tatsache, daB bei Einwirkung von linear polarisiertem Licht auf bestimmte gefärbte Medien diese in der Schwingungsrichtung der Strablung bevorzugte Ejgenschaften erhalten, sie werden dichroitisch und doppelbrechend. Gleichzeitig nehmen die Systeme die Farbe des erregenden Lichtes an. Die wejtere Erforschung dieser Erscheinung hat uuu ergeben, dab kein eigentlich photochemischer Vorgang stattfindet, daB der neue Effekt nur in gefärbten kolloiden Systemen auftritt, und daB wir also Farbänderungen disperser Systeme vor uns haben, welche spezifisch von der Welleriänge des erregenden Lichtes und seiner Schwingungsrichtung abhängig sind.

Man kann demnach das wirkende Agens, die erregende Strablung, in der differenziertesten Art nach Intensität, Welienlănge und Schwingungsrichtung dosieren, und dann die Veränderungen des Systems optisch, also mit einem Mittel gleicher Feinheit. untersuchen.

In einer Reihe von Mitteilungen ${ }^{3}$ ) ist die neue Erscheinung vom physikalischen Stand-

9) F. Weigert, Ueber einen neuen Effekt der Strahlung. I. Mitt. Verh. d. Deutsch. physlk. Ges. 21 479 (1919); il. Mitt. ebenda 21, 615 (1919); III. Mitt. ebenda 21, 623 (1919); IV. Mitt. Zeltschr. f. Physik 2, 1 (1920); V. Mitt. ebenda 3, 437 (1921). - Ueber die spezifische Wirkung der polarisierten Strahlung (Ann. d. Plays. 69, 681, 1920). 
punkt aus untersucht worden, da dieser erste Eall, wo eine vektorielle Eigenschaft der Strahlung, die Schwingungsrichtung, sich dauernd in dem Systen! abbildet, auch für die Kenntnis des Mechanismus der Umwandlung von Strahlung in andere Energieformen von Wichtigkeit zu sein scheint. An dieser Stelle sollen die Versuchsergebnisse in ihren Beziehungen zur Kolloidchemie und besonders zur Farbe disperser Systeme diskutiert werden: Hierzu wird es. nötíg sein, den neuen Effekt zuerst zu beschreiben. Dann werden in einer Reihe folgender Mitteilungen die uliramikroskopische Untersuchung der betreffenden lichtemptindlichen Sysleme, einige Folgerungen aut die optische Struktur der heterogenen Komplexe, die kolloidchemische Deutung des Elfektes und einigeBeispiele besprochen werden, welche zeigen; daB die Erscheínung zur Untersuchung verschiedener Probleme anwendbar ist.

\section{\$2. Die Farbenanpassungen der Photochloride.}

Die neuen Erscheinungen stehen in Beziehung $z \mathrm{u}$ den bekannten Farbenanpassungen der sogenannten Photochloride, uber die zunachst einige Angaben gemacht werden sollen.

Es wurde zuerst yon Ritter ${ }^{4}$, beschrieben, dab die einzelnen Spektralgebiete verschieden auf Chlorsilber wirkten, welches vorher in Licht dunkel ge'ürbt war. W. Seebecks) machte dann 1810 die wichtige Entdeckung, daB die Farbe des angelaufenen Hornatlbers deutlich die Farbe des erregenden bunten Lichtes annimmt: In roten Licht erhalt es eine rote, im blauen eine bläuliche Nuance. Diese Erseheinung ist später şehr eingehend untersucht worden. Auf die Geschichte soll hier nisht eingegangen werden, es soll vielmehr nur das Resultat der gewissermaBen abschlieBenden Untersuchungen Wieners? und Reinders? hier mitgeteilt werden, dal die Farben der erregten Photochloride wirkhiche Körperfarben und keine Interferenz, oder Schillerfarben sind, und daB die Photochloride als eine Adsorptionsverbindung von kolloidem Silber und Chlorsilber nufzutassen sind. Die Parbenanpassung ist nach den Anschauungen Wieners durch ein selektives Ausbleichprinzip auch chemisch

7) Vgl. Eder, Gesch: d. Phot. 1905, 96.

g) Vgl. die Literatur: Verh. d. Deutsch. physik. Ges. 21, 479 (1919).

9) $\mathrm{O}$ Wiener, Wied. Ann. 55, 225 (1895).

W Reinders, Zeitschr. f. physik. Chem. 7 , $213,356.677$ (1911). zu deuten. Danach verschwindet in einem Gemisch verschiedener lichtempfindlicher gefärbter Stoffe bei einfarbiger Beleu,htung nur derjenige, welcher gerade diese Farbe besonders absorbiert, die Absorption für dieses Spektralgebiet nimmt also $a b$, es wird verhältnismäßlg stark durchgelassen und das Gemisch nimmt in weißem Licht die Erregungsfarbe an.

Die Feststellungen $\mathrm{Re}$ inders, daB wir in den farb nempfindlichen Photochloriden Systeme vor uns haben, die lediglich aus Chlorsilber und Silber bestehen, lassen gar keinen Raum mshr für eine groBe Anzahl selektiv in verschiedenen Farben absorbierender gefärbter Verbindungen, die auberdem noch sehr merkwürdige Eigenschaften haben mübten; dann aber schlieben die im Folgenden $\mathrm{zu}$ beschreibenden Beobachtungen mit fast vollkommener Sicherheit eine chemische Deutung der Farbenaripassung mit unserer üblichen Auffassung von chemischen Reaktionen ${ }^{9}$ ) aus und verlangen eine physikalische.

Bis jetzt sind die Parbenänderungen derPhotochloride einfach init dem Auge untersucht worden. Eine spektrophotometrische Ergänzung war daher unbedingt erforderlich.

Als Material wurde in allen messenden Versuchen eine gewöhnliche photographische Chlorsilbergelatine-Emulsion, wie sie für die AristoAuskopierpapiere verwendet wird, auf Glasplatien gegossen. (Ann. IIB) ${ }^{9}$ ). Es entstand so eine sehr durchscheinende Schicht, die mit weibem oder blauem Licht bis zur. Blaurotfärbung belichtet wurde. ( Aniaufen“.) Das von der Präparation noch in der Emulsion enthaltene überschüssige Silbernitrat und die organischen Säuren wurden dann durch längeres Wässern ausgewaschen, wobei die: Färbung einen reinroten Ton annahm; die Platten wurden dann mit sehr intensivem Bogenlicht, das eín orangerotes, grünes oder blaues Farbfilter passiert hatte, eine gewisse Zeit bestrahlt (Ann. IIA), bis die betreffende Plattenstelle eipigermabien eine orangerote, grüne oder blaue Farbennuance angenommen hatte. Diese Farbenveränderung durch farbige $\mathrm{Be}$ strahlung soll im Folgenden immer als $\mathrm{Er}$ regung ${ }^{*}$ bezeichnet werden. Die Farbenanpassung geschieht für die einzelnen Farben verschieden schnell und zwar für Rotgelb am

8) Die Mitwirkung von Elektronen soll in einer späteren Mitteilung diskutiert werden.

9) Experimentelle Einzelheiten über die Versuchie wurden zum Teil schon in den auf S. 115 zitierten Mitteilungen beschrieben. "Es soll im folgenden an den betreffenden Stelien kurz auf die trüheren Arbeiten hingewiesen werden. "Ann. II B " bedeutet 2 . B. "Annalen der Physik 63, 681 (1920). Kap. II B". 
schnellster und für Blau am langzamsten Die angelaufene Photochloridschicht wurde vor und nach der farbigen Erregung spektrophotometrisch untersucht.

In der oberen Hälfte der folgenden Tabeile sind die Extinktionen $\left(\mathrm{E}=\log \mathrm{I}_{0} / 1\right)$ einiger Schichten vor und nach ihrer Erregung mit rotgelbem, grünen und blauem Licht wiedergegeben ( $E_{\text {, und }} E_{\mathfrak{n}}$ ), wie sie sich bei Messung mit dem Spektralphotometer von $\mathrm{K}$ ön ig-Martèns für die Quecksilberlinien $579 \mu \mu$ (Gelb), $546 \mu \mu$ (Grün) und $436 \mu \mu$ (Blau) ergaben. In der unteren Halfte ist das Verhältnis der beiden Werte eingetragen.

\begin{tabular}{|c|c|c|c|c|c|c|}
\hline \multirow{3}{*}{ Metfarben } & \multicolumn{6}{|c|}{ Erregungsarben } \\
\hline & \multicolumn{2}{|c|}{ Ro̊tgelb } & \multicolumn{2}{|c|}{ Grün } & \multicolumn{2}{|c|}{ Blau } \\
\hline & $\mathrm{E}_{\mathbf{q}}$ & $E_{n}$ & $\mathrm{E}_{4}$ & $E_{n}$ & $\mathbf{E}_{\mathbf{v}}$ & $\mathbf{E}_{\mathbf{n}}$ \\
\hline $\begin{array}{l}\text { Gelb }(579 \mu \mu) \\
\text { Grün }(546) \\
\text { Blau }(436)\end{array}$ & $\begin{array}{l}1,652 \\
2,410 \\
2,516\end{array}$ & $\begin{array}{l}1,482 \\
2,488 \\
2,974\end{array}$ & $\begin{array}{l}1,328 \\
1,714 \\
1,500\end{array}$ & $\begin{array}{l}1,461 \\
1,606 \\
1,604\end{array}$ & $\begin{array}{l}1,276 \\
1,696 \\
1,568\end{array}$ & $\begin{array}{l}1,650 \\
1,884 \\
1,482\end{array}$ \\
\hline & \multicolumn{2}{|c|}{$E_{n} / E_{\nabla}$} & \multicolumn{2}{|c|}{$E_{n} / E_{q}$} & \multicolumn{2}{|c|}{$\mathbf{E}_{\mathrm{n}} / \mathrm{E}_{\mathrm{q}}$} \\
\hline $\begin{array}{l}\text { Gelb } \\
\text { Grtin } \\
\text { Blau }\end{array}$ & \multicolumn{2}{|c|}{$\begin{array}{l}0,896 \\
1,033 \\
1,182\end{array}$} & \multicolumn{2}{|c|}{$\begin{array}{r}1,102 \\
0,986 \\
1,069\end{array}$} & \multicolumn{2}{|c|}{$\begin{array}{l}1,293 \\
1,112 \\
0,945\end{array}$} \\
\hline
\end{tabular}

Es geht aus den Messungen hervor, daB die Extinktion in allen Pälen in der Erregungsfarbe abgenommen hat, in den anderen erregungsfremden Parben hat sie dagegen zugenommen. Da in allen Fâllen die Extinktion nicht vollständig verschwindet, ist eine richtige Wiedergabe der Farben nicht möglich. Die eigentliche Parbe der Schicht bleibt wie vor der Brregung rötlich. Nur durch Kontrastwirkung erscheinen die rot-, grün- oder blauerregten Stellen der Schicht röilicher, grünlicher und bläulicher als der Grund. (Ann.VII, A.)

Prinzipiell ist aber die Farbenanpassung viel idealer als sie nach der-im Anfang dieses $A b$ schnitts erwăhnten einfachen Ausbleichauffassung zu erwarten war, da nicht nur die Erregungsfarbe selbst leichter durchgelassen wird, sondern für die benachbarten erregungsfremden Farben der Durchgang erschwert wird. Man kann dies als eine Absorptionsverschiebung bezeichnen. (IV $\$ 23$; Ann. VA, VIIl A.)

Eine chemische Deutung für die Farbenanpassung wird dadurch recht unwahrscheinlich, weil die angenommenen Farbstoffe beim Verschwinden nicht nur in ihrem "eigerien $A b-$ sorptionsgebiet schwächer absorbieren müften, sondern die neu entstehenden Stoffe müßten immer in den erregungsfremden Parben eine stărkere Absorption zeigen als die verschwundenen. Diese Bedingung, welche eine gesetzmatige Heziehung zwischen dem Absorptionsspektrum eines verschwindenden und eines entstehenden Stoffes verlangt, und die noch niemals beobachtet wurde, mübte gleichartig für alle angenommenen Parbstoffe gelten.

Ein weiterer Stütze für die physikalischie Deutung der Farben konnte durch den Nachwers geliefert werden, dab die Silbermenge in den erregten Stellen unverằndert geblieben ist. (V, \$30.) Hierzu wurde eine mit rotem Licht erregte Stelle einer Photochloridplatte fixiert, wobei die Aufhellung erhalten blieb, und dann mit dem bekannten Quecksilberchlorid-Ammoniakverstärker das Silber in die schwarze Komplexverbindung übergeführt. Hierbei rerschwand die aufgehellte Stelle volhommen, sie unterschied sich in ibrer Färbung nicht mehr von den unerregten Stellen der Platte, $d$. h. die pro Flächeneinheit vorhandenen Silberteilchen hatten sich nicht vermindert. Es sind also bei der $\mathrm{Er}$ regung auch keine verschwunden, und eine chemische Reaktion hat nicht stattgefunden. Man kann daher sagen, daß die Parben der Photochloride und ihre Verăndeting durch farbiges Licht rein physikalisch durch den $\mathrm{Zu}$ stand des Silbers und seine Veränderung in dem kolloiden Chlorsilber-Silber-System verursacht wird.

\section{Ein neuer Effekt der Strahlung.}

Wenn man die farbige Erregung der Photo. chloridschichten mit linear polarisiertem Licht ausführt, erhalten die vorher isotropen Schichten die Eigenschaften doppelbrechender Kristalle $(1, \S 2)$ : Die. Aus!öschungsrichtungen der anisotropen Schichten liegen in den Schnittinien der Schwingungsebenen des elektrischen und magnetischen Vektors der erregenden. Strahlung mit der Schicht. Sie seien deshalb in der Folge als die $\mathbf{e}$-Richtung und die $m$-Richtung bezeichnet (IV, 19). Gleichzeitig erhält die Schicht dichroitische Eigenschafien. Dies exkennt man am einfachsten, wenn man die Platten mittelst eines Nikols gegen das Licht betrachtet. Man kann dann durch Drehen des Polarisators vor dem Auge zwei Richtungen einstellen, in denen die Schicht ain hellsten und am dunkelsten erscheint. Diese Richtungen entsprechen der e- und m-Richtung. Man kann also nachträgłich durch diese Betrachtungsart die Richtung der Polarisationsebene der $\mathrm{er}$ - 
regenden Strahlung auffinden. Diese ist also photographisch festgehalten worden.

Die neue Erscheinung läBt sich sehr leicht mit den einfachsten Mitteln demonstrieren, so daB man sie sogar als photochemische oder kolloidchemische Praktikumsaufgabe verwenden kann. Hierzu ist es nur nötig, ein gewöhnliches photographisches Auskopierpapier (CelloidinAristo- oder Albuminpapier) im Tageshicht mittelstark anlaufen zu lassen. Man wäscht dann das Papier mehrmals mit Leitungswasser aus, bis es sich nicht mehr durch Chlorsilber trübt, läßt trocknen und exponiert im Sonneu- oder Bogenlicht in einem Kopierrahmen hinter einem Gelbfilter und einem Nikol'schen Prisma, das mit Kork und Wachs senkrecht auf der Glasplatte des Kopierrahmens befestigt ist. Nach

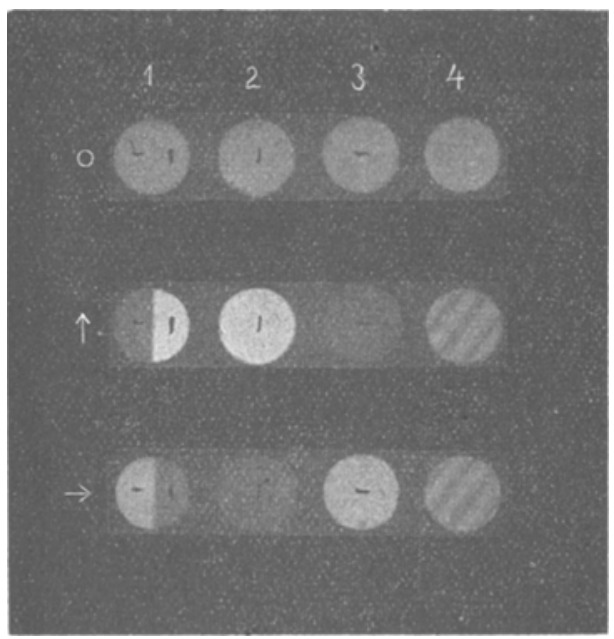

Fig. 1

einiger Zeit hat sich die bestrahite Papierstelle in einem gelblichen. Ton aufgehellt. Beim Betrachten der erregten Stelle in gedämpftem Licht mit einem langsam gedrehten Nikol'schen Prisma erkennt man den Dichroisnus und kann leicht die Polarisatiónsebene des Erregungsnikols durch Aufsuchen der Polarisationsebene des Beobachtungsnikols aufsuchen, bei welcher die erregte Stelle am hellsten erscheint.

Auf den beschriebenen Glasplatten sind die Effekte bedeutender, weil an den weiben Papierflächen durch diffuse Reflexion das polarisierte Licht teilweise depalarisiert wird. Einige Beispiele für starke Effekte sind in der Fig. $1^{10}$

10) Die Klischees für die Fig. 1 und Fig. 2 wurden von Herm Prof. Goetz von der ,Staatl. Akademie für graphische Künste und Buchgewerbe" in Leipzig hergesteilt, dem ich an dieser Stelit meinen verbindlichen Dank aussspreche. wiedergegeben, welche auf folgende Weise entstanden ist (IV, $\S 19$; Ann. II A). Es wurden auf einer Photochloridplatte nebeneinander vier Felder mit polarisiertem roten Licht erregt. Feld 2 mit senkrechter Schwingungsrichtung. Feld 3 mit horizontaler. In Feld 1 stie $B$ ein senkrecht polarisiertes halbkreisförmiges Feld dicht an ein ebensolches horizontal polarisiertes an (Ann. II A) und in Feld 4 wurde in den Strahlengang ein Quarzkeil eingeschaltet (II, \$10). Die vier Felder wurden dreimal auf eine photographische Trockenplatte kopiert, und zwar in der obersten Reihe mit natürlichem, in der zweiten und dritten mit senkrecht und horizontal polarisiertem Licht. Während im ersten Fall alle Felder gleich erscheinen, ist bei der Photographie mit polarisiertem Licht der Dichroismus sehr deutlich. Im vierten Feld erscheinen die bekannten Interferenzstreifen, die ein Quarzkeil zwischen gekreuzten Nikols im Polarisationsmikroskop zeigt. Die hellen und dunklen Streifen haben in der zweiten und dritten Reihe ihre Platze vertauscht.

Eine grobe Reihe von Einzelbeobachtungen ist in den zitierten Abhandlungen beschrieben. Sié erlauben schon jetzt einen allgemeinen Ueberblick über die Veränderungen der lichtempfindlichen Systeme durch die polarisierte Bestrahlung, trotzdem die Erscheinung noch nicht für quantitative Präzisionsversuche reif $z u$ sein scheint (Ann. VII). Dies ist ohne weiteres klar, wenn man bedenkt, dab die Systeme, welche zur Untersuchung kommen, physikalisch sehr kompliziert sind, und daß ihre Vorgeschichte vor Einwirkung der polarisierten Strahlung so unübersichtlich ist, daß schon die Herstellung von mehreren Schichten mit genau reproduzierbaren Eigenschaften unmöglich ist. Es liegen in den Chlorsilber-Gelatine-Emulsionen, den Ausgangsstoffen für diese Versuche; kolloide Systeme vor, deren Eigenschaften sich mit der Zeit, mit der Temperatur, im flüssigen, galleriartigen und trockenen Zustand dauernd in einer Weise ändern, deren wissenschaftliche Erforschung noch im ersten Anfangsstadium steht. Es sei hier nur an die Fabrikation der photographischen Trockenplatten erinnert, welche auch heute noch als eine vollkommen empirische Technik zu bezeichnen ist, trotzdem ungeheuer viel wissenschaftliche Arbeit auf dieses Gebiet verwendet wurde.

Für unseren speziellen Fall kommt dann noch das "Anlaufen hinzu, welches in einer Färbung der Schicht durch metallisches Silber besteht und welche alle zunächst noch unbe- 
kannten Faktoren in sich schlieBt, deren Erforschung der eigentliche Zweck der Untersuchung ist, da ja gerade die Veränderung dieser Färbung durch die Nachbelichtung mit farbigem Licht studiert werden soll.

Man muB datier auf eine absolute Untersuchung der Erscheinungen, welche die Veränderungen in den lichtempfindlichen Systemen in Beziehung zu der absorbierten Strahlungsenergie setzt, zunächst noch verzichten, da derartige Messungen nur dann einen Wert haben, wenn man ihre Bedingungen jederzeit reproduzieren karn. Dagegen sind schon 'jetzt quantitative relative Beobachtungen in der Weise durchführbar, daß man die Veränderungen einer und derselben Stelle einer lichtempfindlichen Schicht bei verschiedenartigen Belichtungen und Nachbehandlungen studiert, oder möglichist die Veränderungen von $z$ wei benachbarten Stellen vergleicht, welche annähernd dieselbe Vorgeschichte haben ").

\section{Der Dichroismus.}

Die Versuche haben besonders interessante Ergebnisse gebracht, als die Wirkung der verschiedenen Erregungsfarben auf Photochloridschichten in den einzelnen Spektralgebieten untersucht wurde. Sie wurden in der Regel so ausgeführt, dat eine Stelle der licbtempfindlichen Schicht mit farbigem polarisiertem Licht erregt und die Veränderung ihrer Eigenschaften von Zeit zu Zeit gemessen wurde. Diese bestand vorwiegend darin, daB die Lichtabsorption sich in der Schwingungsrichtung des elektrischen Vektors (der e-Richtung) anders änderte als in der $\mathrm{m}$-Richtung, in der der magnetische Vektor schwingt. Das System wird also dichroitisch und aus der Messung der in der eund $\mathrm{m}$-Richtung durchgelassenen Lichtintensithlen konnte man diesen Photo-Dichroismus quantitativ bestimmen.

Die Ausführung der Messungen ist in der Arbeit in den Annalen der Physik (Ann.VI, A)

11) $\mathrm{Zu}$ solchen experimentellen Beschränkungen wird man leider hăufig bei der Behandlung photochemischer und kolloidchemischer Probleme gezwungen und besonders ist dies der Fall, wenn beide vereinigt sind, wofür die ganzen photographischen Probleme und auch das hier interessierende Beispiele sind. Die Schlüsse aus den Versuchen bedürfen daher einer vorsichtigen Diskussion und können durch spătere Ergebnisse weitgehend modifiziert werden. Man befindet sich in der Photochemie und in der Kolloidchemie noch auf einem unsicheren Boden und die absolut quantitative Basis, auf der seit einigen Jahren die Atom- und Molekulartheorie aufbauen kann, ist noch ein unerfullter Zukunftswunsch. auŝführlich beschrieben. Hier sei nur mitgeteilt, $\mathrm{daB}$ als quantitatives $\mathrm{MaB}$ für den Dichroismus der Ausdruck $\log I_{e} / I_{m}$ eingeführt wurde, in "welchem $l_{0} / l_{m}$ das Verhältnis der Lichtschwächungen bedeutet, wenn die Strahlen irgend einer Me日-Lichiquelle in der e- oder in der m-Richtung die polarisiert erregte Schicht durchsetzen. Ebenso wie die im vorhergehenderi Kapitel beschriebenen Absorptionsmessungen in den drei Farben Gelb, Grün und Blau des Quecksilberbógens ausgeführt wurden, wurden in diesen Farben und auch im Rot dichrometrische Messungen gemacht und sie ergaben, daß analog zu den Absorptionsverschiebungen durch die farbige Belichtung auch dichroitische Verschiebungen existieren, wenn die Schicht mit polarisiertem Licht erregt war.

Der Aufhellung in der Erregungsfarbe im natürlichen Licht entspricht dann ein positiver Wert von $\log I_{\theta} / I_{m}$, also eine relativ gröbere Aufhellung in der e-Riphtung, einer $A b$ sorptionsverstärkung in den erregungsfremden Farben ein negativer Dichroismus, der sich in einer stärkeren Absorption in der eRichtung ausdrückt. $\mathrm{Da}$ im Anfang bei der Untersuchung immer nur positive Werte des Dichroismus beobachtet wurden, die, wie wir später sehen werden, unmittelbar mit der Erregungsfarbe verbunden sind, wurde er auch al; der normale Effekt bezeichnet, der negative Dichroismus als der inverse Effekt (IV § 27, Ann. V, VI).

Qualitativ ist der Unterschied aus der Fig. 2 zu ersehen (Ana. V, B). Sie enthält einige Spektralaufnahmen des Quecksilberbogens, welche mit einem großen Spektrographen, dessen Spalt breit eingestellt war, gemacht worden sind. $\mathrm{Da}$ die Linien des Quecksilberbogens' sehr weit auseinander stehen, erscheinen sie auch mit dem breiten Spalt noch getrennt. Vor dem Spalt wurde nun eine zur Halfte horizontal und vertikal gelbrot erregte Plattenstelle mit horizontaler Trennungslinie befestigt ( $\mathrm{Nr} .1$ in Fig. 1 , Ann. II, A), und da ja ein Linienspektrum nichts anderes ist als eine Reihe nebeneinander liegender monochromatischur Spalthilder, erscheinen in den Linien die Bilder des vor dem Spalt befindlichen Feldes in den Farben des Quecksilberbogens. Das oberste Spektrum wurde mit natürlichem Licht aufgenommen und zeigt die Absorptionsverschiebung von $\mathrm{S} .117$ in der Aufhellung im Gelb und Grün und derDunklung vom Blau bis Uitraviolett. Die drei anderen Spektren wurden mit linear polarisiertem Licht unter Bedingungen photographiert, die in der 
Figur verzeichnet sind. Man sieht deutlich den normalen. Effekt im Gelb und Grün (das wegen der großen Lichtabsorption nicht sehr stark herausgekommen ist) und den inversen Effekt vom Blau bis Ultraviolett. Die Wirkung ist aber bei $\mathrm{Hg} 334 \mu \mu$ kaum mehr merklich, was damit im Zusammenhang steht, daß das Silber in diesem. ultraviojetten Gebiet ein Absorptionsminimum hat.

Die quantitativen dichrometrischen Messungen lassen nun die Verhältnisse sehr viel weitgehender übersehen, als diese qualitativen photographischen. Darstellungen, vor allen Dingen,
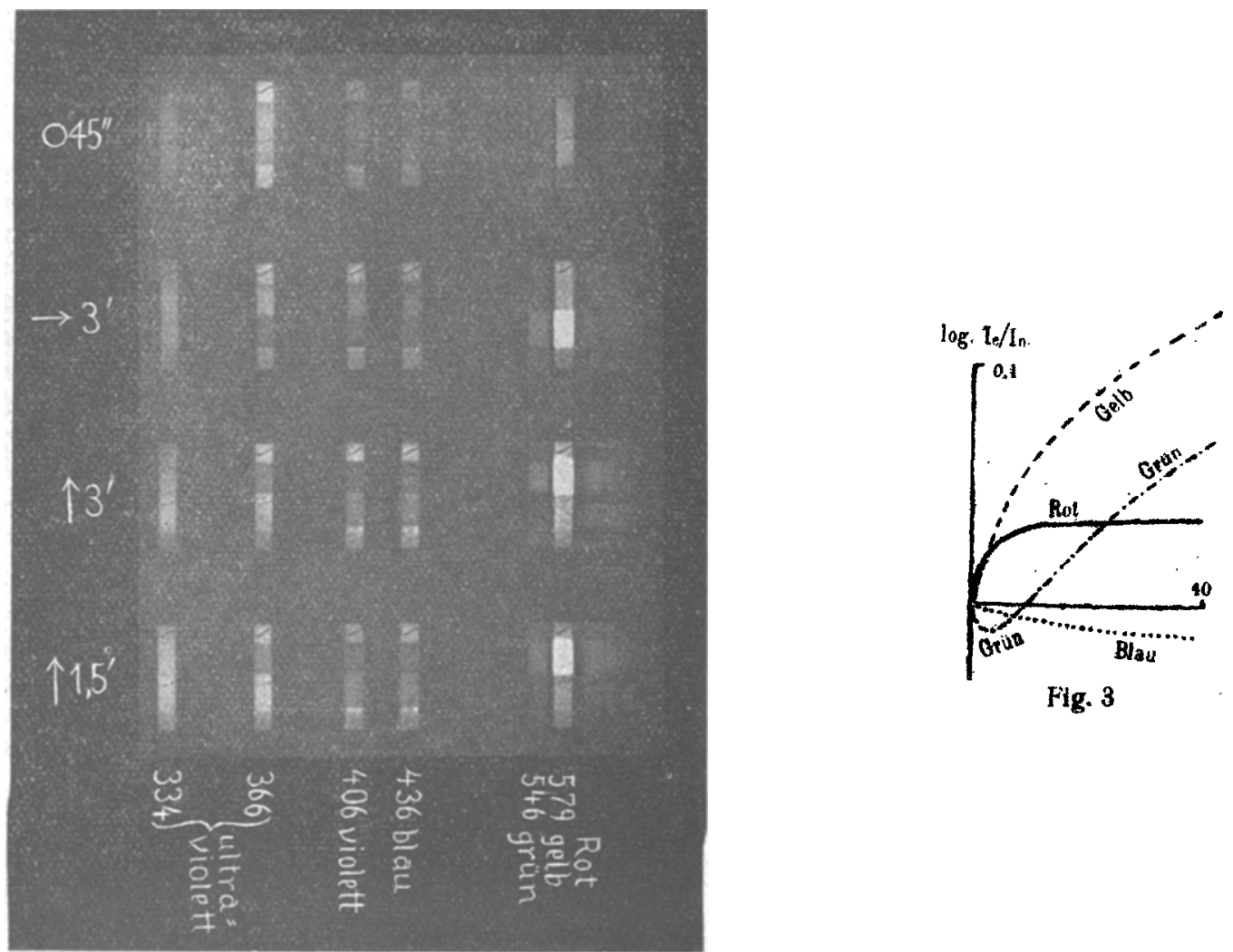

Fig. 3
Die Kurven bedeuten die Veränderungen des Dichroismus in den verschiedenen Mebfarben. Pür diese Ergebnisse gelten selbstverständlich die Beschränkungen von S.119 u. Anm.11, ihrVerlauf ist aber für die verschiedenen Erregungs- und Meffarben ein so typischer und wurde qualitativ in derselben Weise immer wieder gefunden, daB man berechtigt ist, aus ihnen einige allgemeine Schlüsse abzuleiten.

Am charakteristischsten sind die dichrometrischen Veränderungen bei Roterregung (Figuren 3, 4). Wahrend der Dichroismus für Rot sofort steil positiv ansteigt, hat die Kurve

FIg. 2

weil sie noch so feine Unterschiede in den dichroitischen Eigenschaften der Schichten enthüllen, wie sie weder mit dem Auge und noch viel weniger mit der erheblich unempfindlicheren photographischen Platte erkannt werden konnten (Ann. VI, B).

Die Resultate sind in den Figuren $3-7$ graphisch wiedergegeben (IV \& 24; Ann. VII, A). Auf der Abszissenachse ist die Erregungsdauer in Minuten und auf der Ordinate der Dichroismus mit seinem richtigen Vorzeichen afgetragen. 
negativen Aniang hindeutet, geht aus Fig. 5 hervor, in der die Veranderungen bei ganz kurzen Belichtungen dargestellt sind.

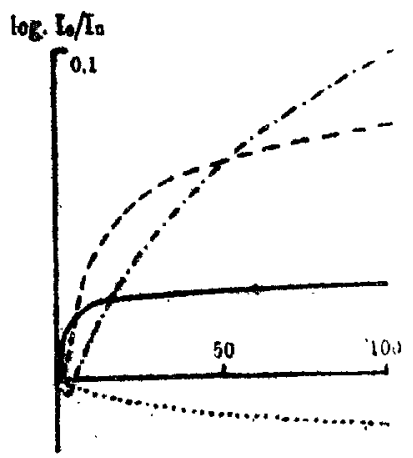

Fig. 4

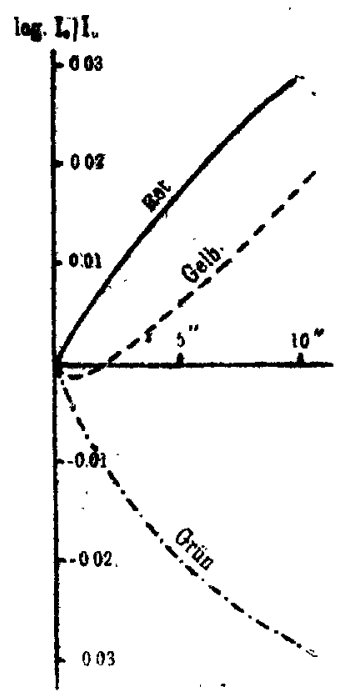

Fig. 5

Der Maßstab von Fig. 5 ist vergrößert, um die feineren Anfangswerte eintragen zu können und man sieht deutlich, wie in den ersten 2 Sekunden auch der Gelbdichroismus negativ ist.

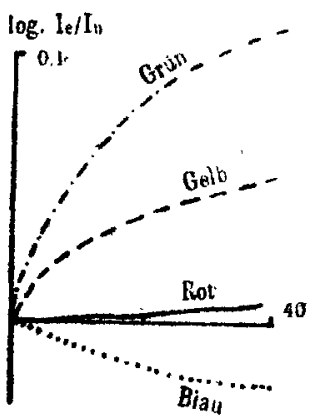

Fig. 6

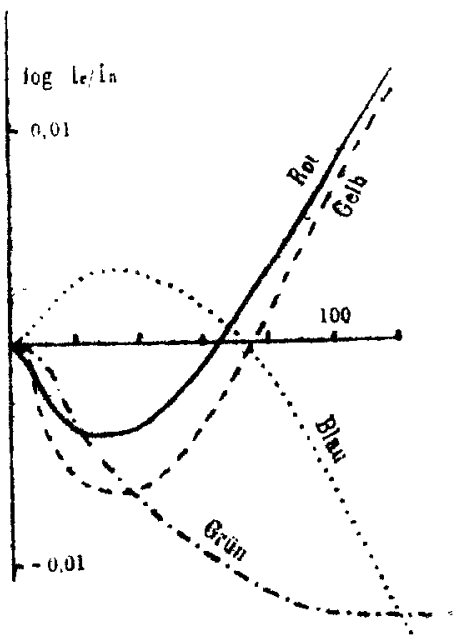

Fig. 7

Die dichroitischen Kurven für Grünerregung sind in Pig. 6 dargestellt. Auch hier ist der Anstieg für die Erregungsfarbe Grün am schăristen, a ăhrend er für die erregungsfremden Farben Gelb, Rot und Blau teils S-förmig, teils ausgesprochen negativ einsetzt. Die Blauerregung endlich, welche in Pig. 7 dargestellt ist, zeigt im Beginn einen positiven Anstieg für Blau und negative Werte für alle anderen Meisfarben. Bei länger dauernder Bestrahlung wechseln aber alle Kurven ihre Richtung: Blau wird negativ; Rot und Gelb positiv, und Grün beginnt sich nach der positiven Seite zu krümmen.

Das allgemeine Ergebnis aller dichrome trischen Messungen an den mit einfarbigem polarisiertem Licht erregten Photochloridgelatineschichten ist folgendes:

Im Beginn der Erregung wird die Schicht für die Erregungsfarbe positiv dichroitisch, in allen erregungsfremden Farben dagegen ist der Effekt entweder ausgesprochen invers oder der Anstieg der Kurven. deutet auf eine anfängliche Negativierung hin. Im Verlauf einer länger dauernden Erregung wechselt der Dichroismus sein Vorzeichen. Dies ist besonders ausgesprochen bei Roterregung für die erregungsfremden. Farben. (Ann. VIli.)

Wenn die polarisiert erregten Schichten einer chemischeri Verstärkung mit Quecksilberchlorid und Ammoniak unterworfen wurden, wie die natürlich erregten am Schlub des $\S 2$, verschwindet der Dichroismus und jede gerichtete. Wirkung vollständig. Dieses Resultat führt zu denselben Schlubfolgerungen wit: früher, daB bei der Erregung die Silbermenge unver- 
ändert geblieben ist, daB also keine eigentliche chemische Reaktion stattgefunden hat, (V. $\$ 30$.)

\section{\$5. Die Dappelbrechung.}

Es wurde schon in den einleitenden Worten des $\$ 3$ mitgeteilt, daß mit dem Dichroismus eine Doppelbrechung der Schicht Hand in Hand geht. Dies folgt ohne weiteres aus der engen Beziehung zwischen Absorption' und Brechungsexponent. Die Schichten erhalten also die Eigenschaften doppelbrechender Kristalle. Wir haben allerdings hier sehr komplizierte optische Systeme vor uns, wenn wir uns erinnern, daß eine gespannte Gelatineschicht schon von vornherein optisch einachsig ist und dab ihre optische Achse in der Richtung der Schichtnormale liegt. Wenn jetzt durch die linear polarisierte Erregung eine neue bevorzugte Richtung

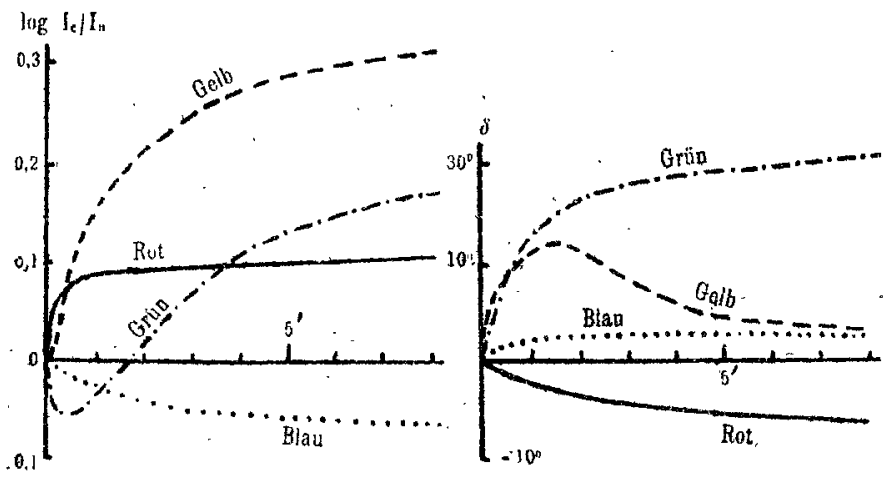

Fig: 8

in der Schichtebene hinzukommt, resultieren optisch zweiachsige Gebilde, die ziemlich schwierig $z \mathfrak{z}$ analysieren sein werden. (Ann. IV, B.) Um diese Verhältnisse aufzuklären, müBte der Effekt senkrecht zur Strahlenrichtung bei der Erregung beobachtet werden. - (IV, $\$ 20$, Ann. IV.) Es konnte nun bei der. Untersuchung dieses Transversaleffektes von der Tatsache Gebrauch gemacht werden, dab der Effekt auch in feuchten gequollenen Gelatinesystemen bei polarisierter Bestrahlung auftritt. $(1, \S 3)$. Diese lassen sich bei vorsichtigem Arbeiten praktisch spannungsfrei und iso'rop herstellen, und so wurde festgestellt, daf in diesem Fall optisch einachsige Gebilde resultieren, deren optische Achse in der Richtung des elektrischen Vektors der erregenden Strahlung liegt.

Nach den Regeln der Kristallphysik schwingt in einem doppelbrechenden einachsigen Kristall der aukerordentliche Strahl in der Richtung der optischen Achse, und der ordentliche senkrecht $z u$ dieser Richtung. In unserem Fall würde dies sagen, daß der auBerordentliche Strahl in der e-Richtung schwingt und der ordentliche in der $\mathrm{m}$-Richtung. Da die Verhältnisse in den trockenen Schichten ganz analog sind, wie in den gallertartigen spannungsfreien, wenn das Licht die Schicht senkrecht durchsetzt, gilt für diese dasselbe.

Da jetzt die Lage der optischen Achse in der. Schicht bekannt ist, kann man auch den Charakter der Doppelbrechung in den photoanisotropen Systemen bestimmen. In der Kristalloptik bezeichnet man einen Kristall als positiv, wenin der Brechungsexponent des auBerordentlichen in der optischen Achse schwingenden Strahles gröber ist, als der des ordentlichen, und in umgekehrten Fall als negativ. Man bestimnt den Charakter und den Grad der Doppelbrechung mittelst eines Kompensators.

Das. gelingt auch bei unseren Schichten und die Ergebnisse bei zunehmender Erregungsdauer sind in den rechten Hälften der Figuren 8 und 9 graphisch eingetragen, während die linken die gleichzeitig aufgenommenen dichrometrischen Kurven enthalten. (IV $\S 25$, Ann. VII B.)

Bei Roterregung ist die Rotkurve dauernd negativ und die Blaukurve dauernd positiv. Die Gelb- und Grünkurve steigen im Anfang nach positiven Werten an. Gelb erreicht schon ziemlich bald ein Maximum und sinkt dann zu niedrigeren positiven Werten herab. Bel sehr langen $\mathrm{Er}$ regungen wendet sich auch Grün um, und Gelb und Grün können sogar negativ werden. Durch Vergleich mit der an derselben

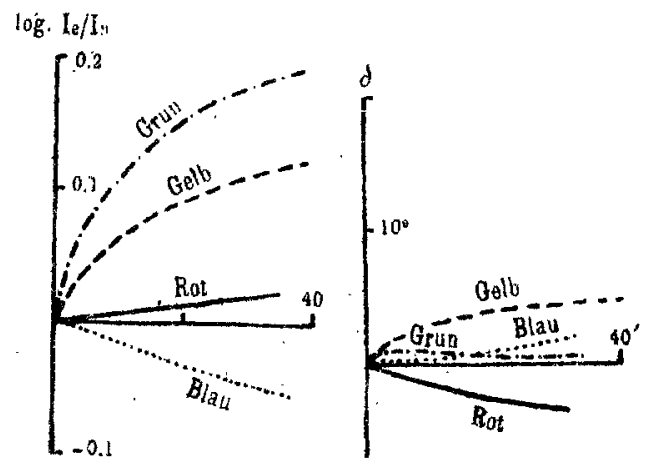

Fig. 9

Schicht aufgenommenen dichrometrischen Kurvenschaar sieht man aber, daß die Vorzeichen und die Lage der Maxima und Minima bei beiden Erscheinungen in keiner einfachen Be- 
ziehung stehen. Nur für Rot und Blau sind immer tie Vorzeichen des Dichroismus und der Doppelbrechung enigegengesetzt. Pür diese beiden Farben gilt also die Babinet'sche Regel, welche aussagt, daß der stärker gebrochene Strahl in einem-doppelbrechenden System auch der sfärker adsorbierte ist. Für die beiden anderen Parben sind die Beziehungen scheinbar ganz regellos.

Bei Grünerregung, für welche der Dichro-. ismus. und die Doppelbrechung in der Figur 9 zusammengestellt sind, sind die Veränderungen șehr viel schwächer und daher noch nicht vollstằndig zu äbersehen, bei Blauerregung endlich konnten überhaupt keine merklichen Doppelbrechungen gemessen werden.

\section{$\$ 6$. Die Empfindlichkeitsspektren.}

Die Abschwächung der Effekte mit abnehmender Wellenlänge sind für die Doppelbrechung und den Dichroismus sehr charakteristisch. Die quantitative Untersuchung der Abnahme der gerichteten Wirkungeń mit der Frequenz der Strahlung, also die Ermittlung ihres Empfindlichkeitsspektrums ist noch nicht abgeschlossen. Qualitativ läBt sich aber schon jetzt mit Sicherheit sagen, daB das Empfindlichkeitsspektrum nicht parallel mit dem $\mathrm{Ab}$ sorptionsspektrum verläuft. Ueber den Verlauf der Absorption in einigen Schichten gibt die Tabelle auf S.117 Auskunft. Die Extinktion $\left(E_{\mathrm{v}}\right)$ ist im Grün und Blau ungefähr gleich stark und sie nimmt äber Gelb nach Rot sehr scbnell ab, so dab die Farbe der Schichten immer eine rote ist.

Selbst hinter einem dunkelroten Glasfilter bilden sich bei polarisierter Erregung sehr schnell dichroitische Ef ${ }^{i}$ ekte aus. Dieses rote Licht wird aber von den -Schichten nur sehr wenig adsorbiert. Im Grün, wo die Absorption sehr stark ist, ist der Effekt geringer als im langwelligen Gebiet, und endlich im Blau ist er nur mit den empfindlichsten Methoden festzustellen. Hand in Hand mit diesen dichroitischen Wirkungen im polarisierten Licht gehen die Aufhellungen und Absorptionsverschiebungen im natürlichen Licht, auf die schon im $\$ 2$ hingewiesen wurde.

Ein scharfer Vergleich der Wirkungen in den verschiedenen Spektralfarben ist sehr schwierig, da die Erscheinung $j x$ in allen Fällen qualitativ verschieden verläuft. Doch kann man schon jetzt sagen, daß die gerichteten Effekte besonders an der langwelligen Seire der $\mathrm{Ab}$ sorptionsbande besonders ausgesprochen sind und auch in den Spektralgebieten noch sebr merklich sind, wo die Absorption selbst nur gering ist. Die Verhälınisse lassen sich am einfachsten an Hand der Fig. 10 übersehen, in welcher die ausgezogene Kurve der Extinktion einer Photochloridschicht entspricht und die gestrichelte einer geschätzten Empfindlichkeit der gerichteten Effekte $(\mathrm{V}, \S 33)$.

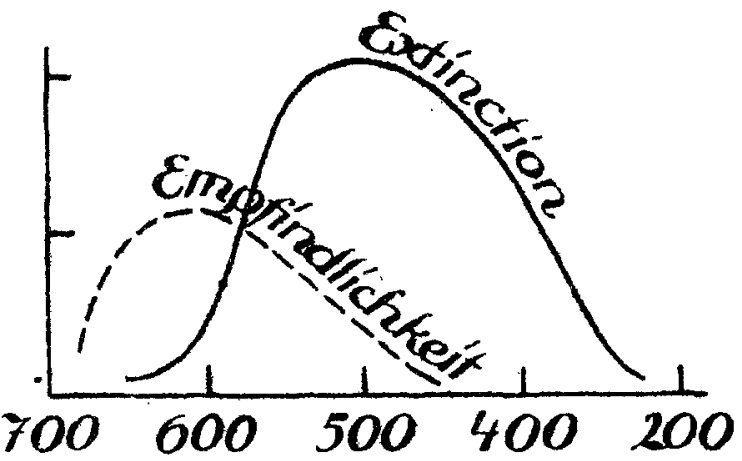

Fig. 10

\section{\$7. Der neue Effekt in Farbstoff. schichten.}

In den vorigen Abschnitten wurden die Versuche beschrieben, welche zur Erforschung der Farbenanpassungen und des gerichteten Effektes der polarisierten Strahlung an PhotochloridGelatineschichten ausgeführt wurden. Es konnte aber auch festgestellt werden, dak die neuen Erscheinungen nicht nur auf diese Systeme beschränkt sind, sondern daB sie auch in ganz anderen Schichten auftreten (I, $\S 6 ; \mathrm{II}, \S 12)$.

Am empfindlichsten erwiesen sich nach den bisherigen Versuchen die lichtempfindlichen Cyaninfarbstoffe in Kollodiumschichten. Wenn man eine trockene Cyanin-Kollodiumschicht auf Glas mit linear polarisiertem weiß̧en oder farbigen Licht erregt, bleicht der Farbstoff aus und die erregte Stelle erscheint bei Betrachtung mit einem Nikol'schen Prisma schwach dichroitisch; die ausgeblichene Stelle erscheint heller; wenn die Polarisationsebenen des Erregungsund Betrachtungsnikols parallel sind, als wenn sie gekreuzt sind. Es entspricht dies in der früheren Bezeichnungsweise einem normalen Effekt oder einem positiven Dichroismus.

Die dichroitischen Verănderungen sind viel geringer als bei den Photochloriden, aber wie bei diesen gehen auch bei den FarbstoffKollodiumschichten deutliche Doppelbrechungen mit ihnen Hand in Hand. Diese lassen sich qualitativ leicht zwischen gekreuzten Nikols in einem Polarisationsmikroskop feststellen, wenn 
taan mögitchst helles Licht -zur Beleuchtung verwendet. In der Diagonallage der erregten Stelie auf dem Objekttisch des Mikroskops, d. h. wenn die e- und $\mathrm{m}$-Richtung die Schwingungsrichtungen der gekreuzten Nikols halbieren, beobachtet man sehr aufiallende Farbenerscheinungen (II, $\$ 12$ ), welche je nach der Erregungsart verschieden sind. Sie treten übrigens unter diesen Bedingungen auch bei den Photochloriden auf (Ann.II, C) und sind durch die optische Aktivität des Kollodiums und der Gelatine verursacht (Ann. VIII, C). Diese anomalen Farbeneffekte sind ein sehr empfindiches qualitatives $\mathrm{Er}$ kennungsmittel für den neuen Effekt.

In den Parbstoffsystemen sind die Effekte viel verganglicher als in den Photochloridgelatineschichten. Sie nehmen schon nach wenigen Tagen an Stärke merklich $a b$, während die dichroitischen Effekte in den Photochloridgelatineschichten noch nach Jahren sehr deutlich sind. Sie sind vor allen Dingen sehr abhängig von dem Zustand des Systems. So treten die dichroitischen Veränderungen überhaupt nicht auf, wenn die Kollodiumschichten nicht vollkommen trocken sind und verschwinden beim nachträglichen Anfeuchten. Daher ist auch die Beobachtung eines Transversaleffektes zur Feststellung der Lage der optischen Achse des photo-anisotropen Systems nicht in den feuchten spannungsfreien Medien möglich wie in der Photochlorid-Gelatinegallerte. Aber durch streifende Erregung konnte gefunden werden, dak auch in den Farbstoffschichten die optische Achse in der Richtung des elektrischen Vektors der Erregungsstrahlung liegt. Auch hier schwingt also der auBerordentliche Strahl in der e-Richtung und der ordentliche in der $m$-Richtung.

Es ist von Interesse, dab das Cyanin in Gelatineschichten den Effekt nicht merklich zeigt, dagegen wurde er bei allen den wichtigen photographischen. Sensibilisierungsfarbstoffen: Pinachrom, Pinazyanol, Pinaverdol,
Orthochrom, Homokoll und Aethylrot in Kollodiumschichten beobachtet. Von anderen Parbstoffgruppen zeigen ihn die substituierten Fluoreszeine, Puehsin, Malachitgrün, Methylviolett und Methylenblau mehr oder weniger deutlich.

Die systematische Untersuchung weiterer Körperklassen in verschiedenen Medien auf das Auftreten des neuen Effektes bei polarisierter Erregung befindet sich erst in den ersten Stadien. Dasselbe gilt auch für die quantitative Messung der zum Teil sehr merkwürdigen Erscheinungen. Die Verhältnisse sind hier erheblich verwickeiter als bei den Photochloriden, weit neben den gerichteten Effekten ein wirklicher chemischer Vorgang, die Ausbleichung, einhergeht. Doch lassen schon die ersten Ergebnisse der quantitativen Untersuchung erkennen, dab sie einen Einblick in den Mechanismus photochemischer Vorgänge erlauben. Sie sollen an anderer Stelle im Zusammenhang mitgeteilt werden. Dort sollen auch einige schwache Farbenanpassungen beschrieben werden, welche an den Parbstoffsystemen bei Erregung mit farbigem natürlichen und polarisierten Licht beobachtet wurden und für welche vor langer Zeit schon Gar.bas so ${ }^{12}$ ) Andeutungen aufgefunden hat (Ann. IX).

An dieser Stelle interessieren die beschriebenen Veränderungen der Farbstoffschichten durch linear polarisiertes Licht vorwiegend wegen ihrer Analogie mit den eingehender stixdierten Photochlorid-Systemen. Auch in diesen Fällen haben wir- typisch kolloide gefärbte Systeme vor uns und die schon bei qualitativer Beobachtung erkannte grobe Empfindlichkeit gegen äuBere Einflüsse spricht für einen sehr engen Zusammenhang zwischen dem Zustand des dispersen Systems und den Verânderungen der Parben im Licht.

12) Garbasso, Niov. Cim. [4] 8, 263 (1898).

\section{Koagulation und Dissolution von Bromsilbersolen durch Ammoniak. \\ (Eingegangen an 26. November-1920.)}

Von Rudolf Auerbach (Probstdeuben b. Leipzig).

Labt man mit der dispersen Phase einer kolloiden Lösung eine chemische Reaktion derart vor sich gehen, daß das entstandene Produkt molekular gelöst ist, so kann das zugesetzte Reagens nicht nur im Sinne kolloid $\rightarrow$ molekular, sondern auch entgegengesetzt durch seine ko- agulierende Wirkung reagieren. Das zeitliche Gesamtresiltat ist also bei gegebener Konzentration durch die Gegrenwirkung von chemischer Auflösungs- und Koagulations-Geschwindigkeit bedingt. Praktisch überwiegt meist einer der beiden Vorgänge und überdeckt dadurch den 